\title{
Photon Counting and Direct ToF Camera Prototype Based on CMOS SPADs
}

\author{
I. Vornicu, R. Carmona-Galán, A. Rodríguez-Vázquez \\ Institute of Microelectronics of Seville (IMSE-CNM), CSIC-University of Seville (Spain) \\ E-mail: ivornicu@imse-cnm.csic.es
}

\begin{abstract}
This paper presents a camera prototype for 2D/3D image capture in low illumination conditions based on singlephoton avalanche-diode (SPAD) image sensor for direct time-offlight $(\mathrm{d}-\mathrm{ToF})$. The imager is a $64 \times 64$ array with in-pixel TDC for high frame rate acquisition. Circuit design techniques are combined to ensure successful 3D image capturing under low sensitivity conditions and high level of uncorrelated noise such as dark count and background illumination. Among them an innovative time gated front-end for the SPAD detector, a reverse start-stop scheme and real-time image reconstruction at $1 \mathrm{kfps}$ are incorporated by the imager. To the best of our knowledge, this is the first ToF camera based on a SPAD sensor fabricated and proved for 3D image reconstruction in a standard CMOS process without any opto-flavor or high voltage option. It has a depth resolution of $1 \mathrm{~cm}$ at an illumination power from less than 6nW/mm² down to $0.1 \mathrm{nW} / \mathrm{mm}^{2}$.
\end{abstract}

Keywords-2D/3D image reconstruction; direct time-of-flight; $S P A D$ image sensor; time gating; low illumination

\section{INTRODUCTION}

Single-Photon Avalanche-Diode (SPAD) image sensors have experienced a tremendous evolution in the last years. The performance of the detector itself has been constantly improved thanks to customized CMOS processes optimized for this kind of photodiodes [1]. Their ability to precisely measure the time-of-flight has fueled a diversity of applications, among them $3 \mathrm{D}$ vision or $3 \mathrm{D}$ ranging. Nowadays smart pixels based on SPADs are becoming a leading technology for ToF imagers. They have been approved even by a very demanding sector such as automotive [2].

When it comes to achieve very high frame rate, an architecture with in-pixel time-to-digital converter (TDC) is more appropriate [3]. Moreover, the integration of 2D imaging by photon counting along with the time resolved functionality has been already proved [4].

Pixel-level depth estimation can be performed by either indirect ToF (i-ToF) or direct ToF (d-ToF) techniques. The former relies on phase difference between the incoming and outgoing continuous or pulsed light-waves [5], [6]. It is less accurate but achieves a higher frame rate and relaxes the requirements of the illumination source. The latter technique directly measures the time elapsed for the light wave to travel from the laser to the target and back to the sensor [7], [8], [9]. It is more accurate but demands picosecond-jitter lasers.

This paper reports a prototype of a SPAD camera for $3 \mathrm{D}$ image reconstruction based on a $64 \times 64 \mathrm{~d}-\mathrm{ToF}$ imager with in- pixel TDC of less than $150 \mathrm{ps}$ time resolution. It also incorporates a $2 \mathrm{D}$ vision capability by photon counting.

The main motivation of this project was to build a SPAD based camera in a cost-effective standard CMOS technology for mixed signal circuit design but with moderate performance for integrated photodiodes [10]. In this case the noise has an average count rate of $42 \mathrm{kHz}$ and a photon detection probability of $6.5 \%$ at $520 \mathrm{~nm}$. Under these circumstances we had to face big challenges to be able to retrieve depth and brightness images.

In order to counteract the weaknesses of the SPAD detector, several techniques have been employed such as: fast active quenching/ recharge circuit, time gating of the SPAD frontend, reverse start-stop. They have a contribution at circuit design level which also helps to reduce the power consumption which is crucial for large arrays of SPADs and in-pixel TDCs. Moreover, embedded real-time image reconstruction at $1 \mathrm{kfps}$ has been implemented off-chip.

By applying the aforementioned design strategies we have been able to reconstruct 2D and 3D images with performances close to the state-of-the-art. The prototype of the camera has also a graphical user interface (GUI) and command-line interface built in Matlab and $\mathrm{C}++$.

The principle of d-ToF is explained in the diagram of the experimental setup depicted in Fig. 1. A picosecond laser sends a short light pulse towards an object plane, which can be at any position between $\mathrm{P}_{\mathrm{i}}$ and $\mathrm{P}_{\mathrm{f}}$. At the same time, an electrical signal is sent to the global shutter of the imager. Some of the outgoing photons reflected by the object reach the active surface of the sensor and are eventually detected.

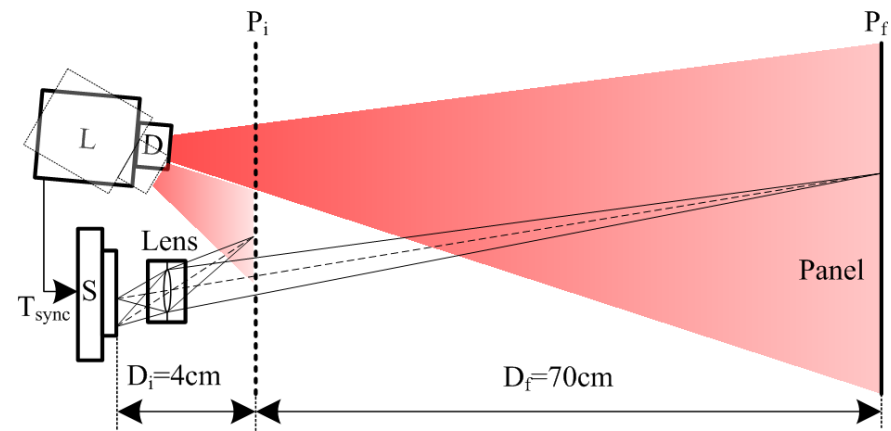

Fig. 1. Top view of the setup for distance measurement and array uniformity. $\mathrm{L}=$ picosecond laser; $\mathrm{D}=$ diffuser; $\mathrm{S}=\mathrm{SPAD}$ image sensor 
The time elapsed between the detection of one photon and the synchronization signal is actually a direct estimation of the time-of-flight, which in turn is proportional to the distance between the sensor and the object. Notice that, in our case, the maximum distance is limited by the available equipment, i.e. optical table dimensions and laser power.

The rest of the paper is organized as follows: Section II introduces the camera prototype and its most innovative features. Section III explains the experimental setup employed for 3D image reconstruction in low illumination conditions. Section IV is dedicated to experimental results, including snapshots of the camera configured both for 2D and 3D vision. Section V draws the conclusions of this work.

\section{PROTOTYPE OF THE SPAD CAMERA}

The d-ToF system is composed by the SPAD camera (Fig. 2 ) and the picosecond laser. The housing of the camera, built by a $3 \mathrm{D}$ printer, accommodates an $8 \mathrm{~mm}$ F1.2 lenses focusing on the $64 \times 64$ array of SPADs and pixel-level TDCs. These lenses perform a proper framing of a $30.5 \times 41 \mathrm{~cm}$ panel placed at $74 \mathrm{~cm}$ from the sensor. The control signals are provided by an FPGA board located below the PCB of the image sensor. It also implements a real time $2 \mathrm{D}$ and $3 \mathrm{D}$ image reconstruction and an USB link used for luminous intensity (2D) and depth (3D) image streaming and some configurations of the camera. A user-friendly GUI has been built in Matlab. The camera also has the possibility to record videos at $1 \mathrm{kfps}$ and sent them to the computer in raw format. Afterwards the user can play them off-line at $50 \mathrm{fps}$.

The camera can be also connected to an in-house designed Time Interval Generator (TIG) of 8ps incremental resolution. In this way the array of TDCs is tested much more efficiently comparing with the amount of data and acquisition time required by the code density test. Using a command-line interface, the user can run a specific test and save the static characteristics of the TDCs.

The SPAD imager has been fabricated in UMC $0.18 \mu \mathrm{m}$ technology. It is composed by the pixel array and some on-chip peripheral circuits such as: PLL-based global compensation loop for Process parameters, Voltage supply and Temperature (PVT) variations, analog buffers for uniform distribution of the input reference voltages for the TDCs, clock tree distribution of the START and STOP signals across the array and a readout back-end which serializes data at $50 \mathrm{MHz}$.

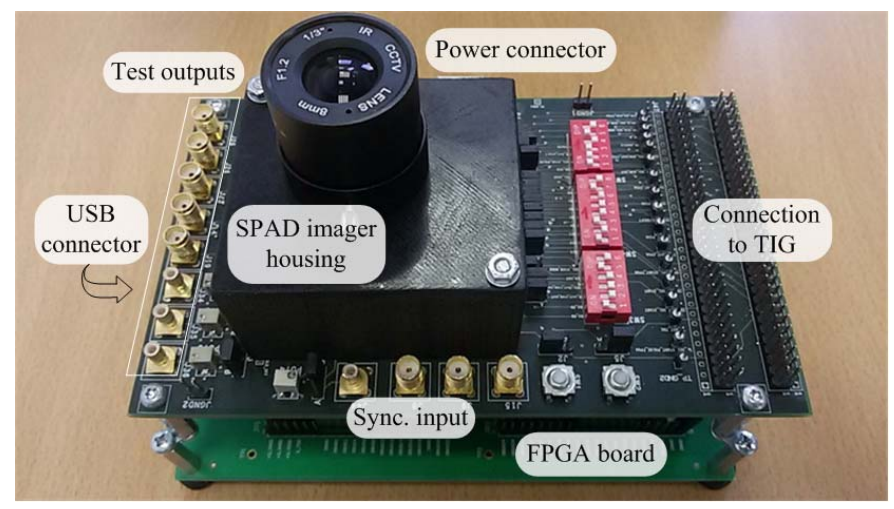

Fig. 2. Prototype of the SPAD camera
This technological limitation can be counteracted by parallelizing the output. Thus the imager can reach a theoretical speed of $32 \mathrm{kfps}$. Each pixel contains the SPAD detector with a fast quenching/ recharge circuit, the start-stop logic, the programmable time bin TDC and a static memory module with output buffers. A time gated SPAD front-end has been integrated in order to mitigate the high level of uncorrelated noise and to reduce power consumption. The start-stop logic block implements the reverse start-stop technique which leads to further decrease of the power consumption. Therefore a TDC can be triggered by a SPAD pulse and turned off by a synchronization pulse. In addition to that it takes into account only the first pulse in the time gate, ignoring all the others. Moreover the sensor integrates the control for the test of the TDC array by means of a TIG embedded in the system. Each TDC employs a Voltage-Controlled Ring-Oscillator (VCRO) as time interpolator such that it has a few nanoseconds conversion time [11].

The most exciting features of this camera are the time-gated pixel and the real-time image reconstruction. The former one has been integrated by a novel time-gated active quenching/ recharge circuit. By playing with the size of the time-gate, we can increase the Signal-to-Noise Ratio (SNR) in photon timing applications. The latter feature is based on pixel level histogram building of a certain number of measurements which from now on will be called inter-frames. There is a trade-off between accuracy and overall frame rate of the reconstructed images [12]. This is because the jitter FWHM of a ToF measurement decreases by the square root of the number of inter-frames. Note that the jitter in this case is mainly due to the jitter of the SPAD detector and the jitter of the TDC. Last but not least, the minimum number of inter-frames to achieve certain accuracy depends also on the Photon Detection Efficiency (PDE) of the detector and the amount of the uncorrelated noise. The specifications of the picosecond laser employed in the measurements are presented in the next sections.

\section{LOW ILLUMINATION SETUP}

In order to acquire depth images by d-ToF in low illumination conditions, we have employed a PicoQuant D-C640 pulsed laser [13] and a Thorlabs ED1-S50 diffuser [14].

This combination outputs a square pattern and guarantees uniform distribution of light over the scene.

The wavelength is $640 \mathrm{~nm}$ with an average output power of $160 \mu \mathrm{W}$, and a repetition rate of $2.5 \mathrm{MHz}$. The pulse width is below 90ps and the jitter of the synchronization signal is $20 \mathrm{ps}$.

The reflected power received by the sensor is measured by using a Newport 918-SL photodetector. The photodetector is coupled with the $8 \mathrm{~mm}$ lenses. The setup diagram is similar with the one depicted in Fig. 1.

The distance between the photodetector and the panel varies in our experimental setup from $88 \mathrm{~cm}$ to $11 \mathrm{~cm}$ with $1 \mathrm{~cm}$ step. The measured irradiance is depicted in Fig. 3. The maximum irradiance is below $10 \mathrm{nW} / \mathrm{mm}^{2}$ which is consistent with the illumination condition for single photon detection. Notice that the distance range in this experimental setup is limited by the power of the laser. 


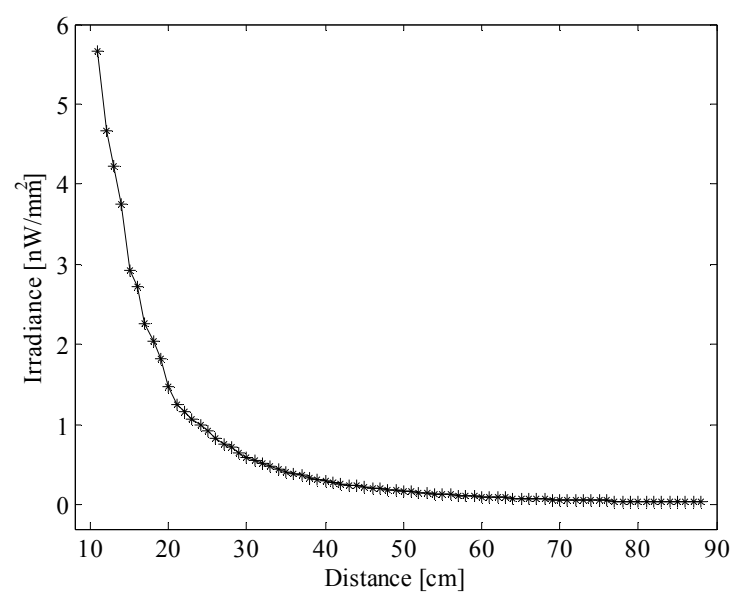

Fig. 3. Measured irradiance of the reflected light

\section{EXPERIMENTAL RESULTS}

\section{A. $3 D$ ranging}

The aim of this experimental setup is to measure the depth resolution, linearity and array homogeneity for short distances. We have employed a panel to provide a homogenous background for the shapes to be reconstructed. This panel has been displaced towards the sensor-laser ensemble from $74 \mathrm{~cm}$ down to $4 \mathrm{~cm}$ with $1 \mathrm{~cm}$ step (see Fig. 1). For better illustration of the spatial linearity, only the closest and farthest depth images are depicted in Fig. 4. Each measurement is obtained by averaging $65 \mathrm{k}$ inter-frames.

Any pixel of the same tier has to estimate the same distance. Therefore the incremental resolution is improved even further by spatial averaging these pixels (see Fig. 5). Notice that in the middle range from $13 \mathrm{~cm}$ to $66 \mathrm{~cm}$ the sensor behaves quite linear, having an incremental depth resolution of $1 \mathrm{~cm}$. The distortion below $13 \mathrm{~cm}$ is caused by the reflections of the lens which is less than $2 \mathrm{~cm}$ close to the panel. These reflections increase the jitter of the SPAD detector, lowering the accuracy of the measurement. The nonlinearities above $66 \mathrm{~cm}$ are due to the low amount of light that reaches the sensor. In this case the sensor is mostly triggered by noise. Therefore in order to achieve the same accuracy as in the middle range, more than $65 \mathrm{k}$ inter-frames would be needed.

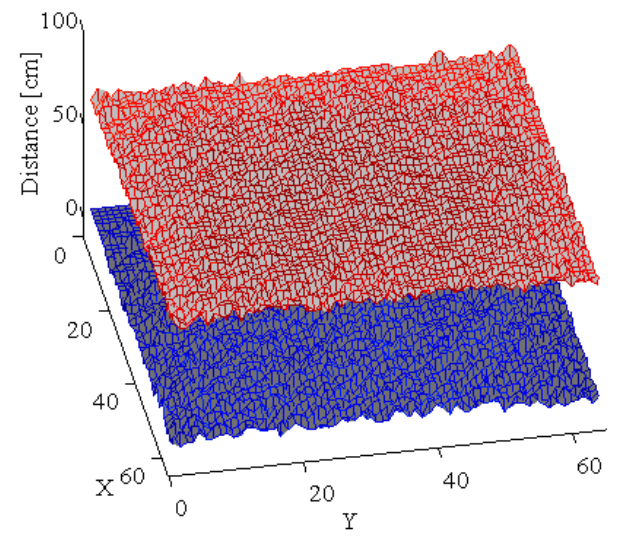

Fig. 4. The panel is placed at $4 \mathrm{~cm}$ (bottom plate) and $74 \mathrm{~cm}$ (upper plate) towards the sensor

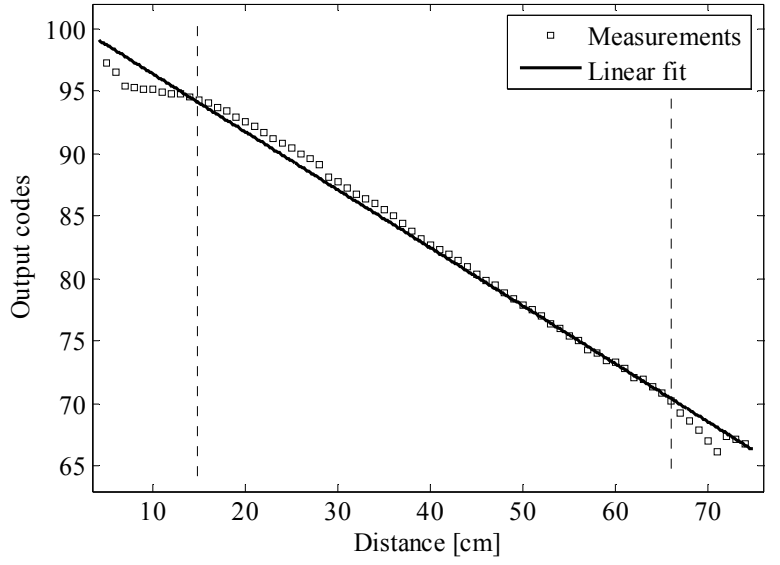

Fig. 5. Distance measurement by averaging all the pixels of the array. The depth resolution is $1 \mathrm{~cm}$.

\section{B. $2 D$ and $3 D$ imaging}

The camera is able to capture $2 \mathrm{D}$ and $3 \mathrm{D}$ images. The experimental setup is depicted in Fig. 6 a). The scene consists of a geometric object $\mathrm{O}_{2}$ placed in front of a white panel located in position $\mathrm{P}_{2}$. The SPAD array is biased at an excess voltage of $1.1 \mathrm{~V}$

The brightness map is obtained by merely counting photons. This is done at pixel-level by a gated $8 \mathrm{~b}$ counter which is actually the coarse counter used by the TDC for $3 \mathrm{D}$ imaging. The depth map is computed from the d-ToF measured by every pixel of the array.

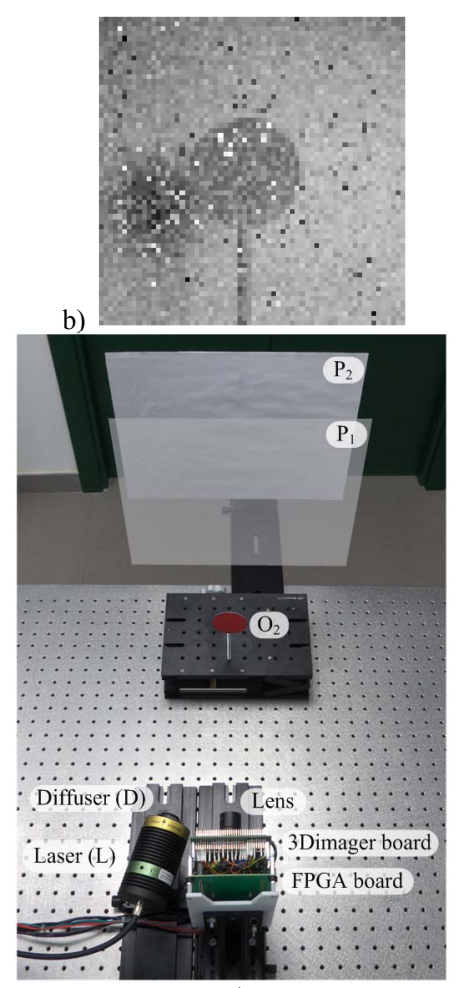

a)
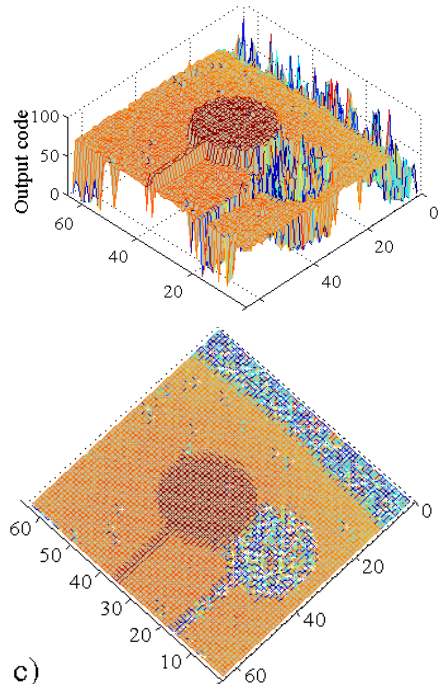

c)

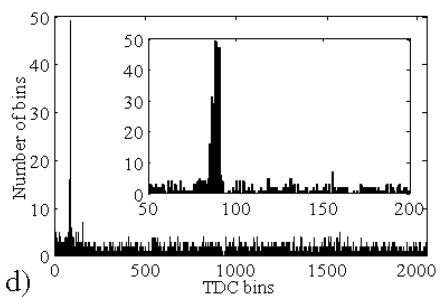

Fig. 6. a) Experimental setup for 2D and 3D imaging; b) 2D shape reconstruction; c) 3D shape reconstruction; d) ToF IRF of pixel $(32,32)$. 
$2 D$ vision: The scene is lighted up with an illuminance of 100 lux. This amount of light is enough to ensure the minimum count rate required at the input of the CMOS counters which are driven by the SPAD detectors. The 2D image obtained by averaging 100 inter-frames is depicted in Fig. 6 b). In this case the frame rate of the reconstructed images is about $10 \mathrm{fps}$. Notice the shadow of the object placed in front of the panel.

$3 D$ vision: The laser is setup as explained in Section III. The raw image is shown in Fig. 6 c) from two angles of view. It is reconstructed from $65 \mathrm{k}$ inter-frames. Even more, it still has a quite good accuracy only with $4 \mathrm{k}$ inter-frames. The pixels from the upper side of the image are out of the panel area while those from the right side of the geometric shape are shaded by it. In both cases the pixels are triggered only by noise since no reflected laser light reaches them back. Moreover, the histogram for the pixel $(32,32)$ is depicted in Fig. 6 d). It is built off-chip at $1 \mathrm{kfps}$, based on the ongoing inter-frames. The SNR of the ToF measurement is about $24 \mathrm{~dB}$. The jitter FWHM is about 735 ps.

Thanks to the reliability of the time-gated and 3D image reconstruction technics employed at circuit and system level, this camera is able to work in $3 \mathrm{D}$ mode even when the uncorrelated noise rate is 20 times larger than the average DCR. In this case at least $40 \mathrm{k}$ inter-frames are required to reconstruct the $3 \mathrm{D}$ image.

A comparison with the state-of-the-art SPAD camera prototypes is performed in TABLE I. As can be seen, the performance of our camera is in the state-of-the-art, despite of the poor sensor characteristics. Thanks to the features highlighted in Section II, our camera is able to build 2D and 3D images even in the harsh condition of low performance SPAD detectors such as a high average DCR of $42 \mathrm{kHz}$ and a low PDE of $5 \%$ at $640 \mathrm{~nm}$.

TABLE I. COMPARISON WITH THE STATE-OF-THE-ART

\begin{tabular}{ccccc}
\hline Performances & {$[6]$} & {$[7]$} & {$[9]$} & This work \\
\hline \hline Technology & $0.35 \mu \mathrm{m}(1)$ & $0.35 \mu \mathrm{m}(1)$ & $0.13 \mu \mathrm{m}(2)$ & $0.18 \mu \mathrm{m}(3)$ \\
No. of pixels & $64 \times 32$ & $32 \times 4$ & $32 \times 32$ & $64 \times 64$ \\
SPAD size & $30 \mu \mathrm{m}$ & $30 \mu \mathrm{m}$ & $15 \mu \mathrm{m}$ & $12 \mu \mathrm{m}$ \\
Fill factor & $3.14 \%$ & $3.14 \%$ & $2 \%$ & $2.7 \%$ \\
Imaging lens & 1.4 & n.a. & n.a & 1.2 \\
Illumination & $800 \mathrm{~mW}$ & $100 \mathrm{nW}$ & $2 \mathrm{~mW}$ & $160 \mu \mathrm{W}$ \\
average power & & & & \\
Illumination & $850 \mathrm{~nm}$ & $555 \mathrm{~nm}$ & $405 \mathrm{~nm}$ & $640 \mathrm{~nm}$ \\
wavelenght & & & & \\
Median DCR & $100 \mathrm{~Hz}$ & $70 \mathrm{~Hz}$ & $100 \mathrm{~Hz}$ & $42 \mathrm{kHz}$ \\
PDE @ $\lambda$ & $5 \% @$ & $30 \% @$ & $25 \%$ & $5 \% @$ \\
In-pixel TDC & $850 \mathrm{~nm}$ & $520 \mathrm{~nm}$ & & $640 \mathrm{~nm}$ \\
architecture & $\mathrm{U} / \mathrm{D}$ & Delay line & Delay line & VCRO \\
TDC res. & n.a. & $400 \mathrm{ps}$ & $119 \mathrm{ps}$ & $150 \mathrm{ps}$ \\
Depth res. & $10 \mathrm{~cm}$ & $1 \mathrm{~cm}$ & $1 \mathrm{~cm}$ & $1 \mathrm{~cm}$ \\
Power/ TDC & n.a. & $6 \mathrm{~mW}$ & n.a & $1.8 \mathrm{~mW}$ \\
ToF technique & $\mathrm{CW}-\mathrm{i}-\mathrm{ToF}$ & $\mathrm{d}-\mathrm{ToF}$ & $\mathrm{d}-\mathrm{ToF}$ & $\mathrm{d}-\mathrm{ToF}$ \\
\hline (1) Very low & & & & \\
\hline
\end{tabular}

(1) Very low defect, customized for SPADs; (2) Customized for CMOS image sensors; (3) Mixed-signal standard technology without any opto-flavor or high voltage option.

\section{CONCLUSION}

This paper presents a camera prototype for $2 \mathrm{D} / 3 \mathrm{D}$ image reconstruction based on photon counting and $\mathrm{d}-\mathrm{ToF}$. Taking into account that the overall frame rate is limited by the required large number of inter-frames and image serialization, we are planning to move the real-time image reconstruction on chip and parallelize the sensor reading.

\section{ACKNOWLEDGMENT}

This work has been funded by the Office of Naval Research (USA) ONR, grant No. N000141410355, the Spanish Ministry of Economy (MINECO) through project TEC2015-66878-C31-R (European Region Development Fund, ERDF/FEDER), and Junta de Andalucía, Consejería de Economía, Innovación, Ciencia y Empleo (CEICE) P12-TIC 2338

\section{REFERENCES}

[1] G.-F. Dalla Betta, L. Pancheri, D. Stoppa, et al., "Avalanche photodiodes in submicron CMOS technologies for high-sensitivity imaging", InTech, Advances in Photodiodes, pp. 225-248, 2011, DOI: 10.5772/15178. Online: http://www.intechopen.com/books/advances-inphotodiodes/avalanche-photodiodes-in-submicron-cmos-technologiesfor-high-sensitivity-imaging

[2] F. Zappa, A. Tosi, "MiSPIA: microelectronic single-photon 3D imaging arrays for low-light high-speed safety and security", Adv. Ph. Count. Tech. VII, Proc. of SPIE, Vol. 8727, 87270L, DOI: 10.1117/12.2018661, 2013

[3] J. Richardson, R. Walker, L. Grant, et al., “A 32×32 50ps resolution 10bit time to digital converter array in 130nm CMOS for time correlated imaging", Custom Int. Circ. Conf., pp. 77-80, 2009.

[4] F. Villa, R. Lussana, D. Bronzi, et al., "CMOS imager with 1024 SPADs and TDCs for single-photon timing and 3-D time-of-flight", J. of Sel. Top. in Quant. Elect., Vol. 20, No. 6, Nov. 2014

[5] S. Bellisai, L. Ferreti, F. Villa, et al., "Low-power, 20 meter 3D ranging SPAD camera based on continuous-wave indirect time-of-flight", Adv. Ph. Count. Tech. VI, Proc. of SPIE, Vol. 8375, 83750E, DOI: $10.1117 / 12.920407,2012$

[6] D. Bronzi, F. Villa, S. Tisa, et al., "100 000 frames/s $64 \times 32$ singlephoton detector array for 2-D imaging and 3-D ranging”, J. of Sel. Top. in Quant. Elect., Vol. 20, No. 6, Nov./Dec. 2014

[7] F. Villa, R. Lussana, D. Tamborini, et al., "CMOS single photon sensor with in-pixel TDC of time-of-flight applications", Nordic-Medit. Workshop on TDC, DOI:10.1109/NoMeTDC.2013.6658230, Nov. 2013

[8] M. Perenzoni, D. Perenzoni, D. Stoppa, "A 64×64-pixel digital silicon photomultiplier direct ToF sensor with 100MPhotons/s/pixel background rejection and imaging/altimeter mode with $0.14 \%$ precision up to $6 \mathrm{~km}$ for spacecraft navigation and landing”, Int. Solid-State Circ. Conf., pp. 118-119, 2016

[9] M. Gersbach, Y. Maruyama, R. Trimananda, et al., "A time-resolved, low-noise single-photon image sensor fabricated in deep-submicron CMOS technology", J. of Solid-State Circuits, Vol. 47, No. 6, June 2012

[10] I. Vornicu, R. Carmona-Galán, Á. Rodríguez-Vázquez, “A SPAD plus TDC array for 145 ps-accuracy ToF measurement". IS\&T/SPIE Elect. Imag.: Image Sensors and Imaging Systems, Proc. of SPIE, San Francisco, California (USA), Vol. 9403, pp. 94030I.1-6, Feb. 2015

[11] I. Vornicu, R. Carmona-Galan, A. Rodriguez-Vazquez, "In-pixel voltage-controlled ring-oscillator for phase interpolation in ToF image sensors", Int. Symp. on Circ. and Systems, pp. 1906-1909, May 2016

[12] I. Vornicu, R. Carmona-Galan, A. Rodriguez-Vazquez, "On the calibration of a SPAD-based 3D imager with in-pixel TDC using a timegated technique", Int. Symp. on Circ. and Sys., pp. 1102-1105, 2015

[13] https://www.picoquant.com/products/category/picosecond-pulsedsources/ldh-series-picosecond-pulsed-diode-laser-heads for PicoQuant D-C-640 pulsed laser data sheet, PicoQuant GmbH.

[14] https://www.thorlabs.com/thorproduct.cfm?partnumber=ED1-S50 for Thorlabs ED1-S50 diffuser. 